\title{
Construcción de la Función Integral y Razonamiento Covariacional: dos Estudios de Casos
}

\author{
Building the Concept of Integral Function and Covariational Reasoning: \\ two Case Studies
}

\author{
Carmen Aranda* \\ María Luz Callejo**
}

\begin{abstract}
Resumen
Este artículo identifica algunas características de cómo estudiantes de Bachillerato (17-18 años) construyen el concepto de función integral en un experimento de enseñanza utilizando applets, y diseñado según una trayectoria hipotética de aprendizaje. De los quince estudiantes que participaron en el experimento de enseñanza se han seleccionado cinco, que se encuentran en distintos momentos del proceso de abstracción reflexiva, para hacer un estudio de casos. Los resultados indican tres características del proceso de construcción de la función integral: (i) no identificar la relación entre un extremo de un intervalo y el valor del área bajo una recta en dicho intervalo; (ii) identificar esta relación como una forma de covariación simple; y (iii) reconocer la covariación compleja entre la variable $\mathrm{x}, \mathrm{x} \in[\mathrm{a}, \mathrm{b}]$, una función $\mathrm{f}(\mathrm{t}), \mathrm{t} \in[\mathrm{a}, \mathrm{x}]$ y la función integral $F(x)=\int_{a}^{x} f(t) d t$, en funciones lineales. Estos resultados aportan información sobre la trayectoria de aprendizaje del concepto de función integral que puede ser usado en el diseño de materiales curriculares que apoyen el razonamiento covariacional en los estudiantes.
\end{abstract}

Palabras clave: Abstracción reflexiva. Experimento de Enseñanza. Trayectoria Hipotética de Aprendizaje. Uso de applets.

\begin{abstract}
The aim of this paper is to characterize how high school students build the integral function concept in the context of a teaching experiment using applets, and designed according to a hypothetical learning trajectory. Five out of fifteen students were chosen, which have participated in the teaching experiment and shown different moments of reflective abstraction process, to carry out a study of cases. Our results indicated three characteristics for building the integral function concept: (i) not identifying the relationship between a bound of an interval and the value of the area under a straight line belonging to this interval; (ii) identifying this relationship as a way of simple covariation; and (iii) identifying the complex covariation among the variable $\mathrm{x}, \mathrm{x}$ $\in[\mathrm{a}, \mathrm{b}]$, a function $\mathrm{f}(\mathrm{t}), \mathrm{t} \in[\mathrm{a}, \mathrm{x}]$ and the integral function $F(x)=\int_{a}^{x} f(t) d t$, in linear functions. These results provide information about the learning trajectory of the integral function concept; this trajectory can be used for designing curricular materials that support students' covariational reasoning.
\end{abstract}

Keywords: Reflexive abstraction. Teaching Experiment. Hypothetical Learning Trajectory. Covariational Reasoning. Integrating applets.

\footnotetext{
* Doctora en Didáctica de la Matemática por la Universidad de Alicante (UA). Profesora de Matemáticas de Secundaria del Instituto de Educación Secundaria (I.E.S), Alicante, España. Dirección postal: Calle Dr Fleming $n^{\circ}$ 3, La Vila Joiosa, CP 03570, Alicante, España. E-mail: carmen.arlo@gmail.com

${ }^{* *}$ Doctora en Didáctica de las Disciplinas, opción Matemáticas, por la Universidad Denis Diderot (Paris 7). Profesora Titular de Didáctica de la Matemática en la Universidad de Alicante (UA). Dirección postal: Carretera San Vicente del Raspeig s/n, CP 03690, San Vicente del Raspeig, Alicante, España. E-mail: luz.callejo@ ua.es
} 


\section{Introducción}

La enseñanza del Cálculo constituye una parte importante de los programas en la enseñanza post-obligatoria (16-18 años) y de algunos estudios universitarios y su aprendizaje ha sido objeto de debate e investigación en las últimas décadas (AZCÁRATE et al., 2015; RASMUNSSEN; BORBA, 2014). El estudio del Cálculo se centra en la comprensión de conceptos vinculados a procesos infinitos, sin embargo, muchas veces su presentación se focaliza en aspectos procedimentales como el manejo de reglas para calcular límites, derivadas o integrales (GONZÁLEZ-MARTÍN; CAMACHO, 2004; THOMPSON, 1994a).

Para comprender los conceptos fundamentales del Cálculo diversas investigaciones han puesto de relieve la importancia del razonamiento covariacional (COTRILL et al., 1996; JOHNSON, 2015; KAPUT, 1992; THOMPSON, 1994b; ZANDIEH, 2000). Este tipo de razonamiento ha sido definido como "las actividades cognitivas implicadas en la coordinación de dos cantidades variables mientras se atiende a las formas en que cambian unas en relación a otras" (CARLSON et al., 2002, p. 354).

El razonamiento covariacional se apoya en cinco actividades de coordinación cognitiva: entre el valor de una variable y los cambios en la otra; entre la dirección del cambio de una variable y los cambios en la otra; entre la cantidad de cambio de una variable y los cambios en la otra; entre la tasa de variación media de una función y el incremento uniforme de la variable independiente; entre la tasa de variación instantánea de una función y el cambio continuo de la variable independiente.

Estas coordinaciones han sido puestas de relieve por Cotrill et al. (1996) al sugerir usar la concepción dinámica para introducir el concepto de límite de una función. Según esta concepción, el límite de una función $f(x)$ es $L$ cuando $x$ tiende a $a$, si cuando $x$ se acerca al número a más que cualquier aproximación, sus imágenes $f(x)$ se acercan a $L$ más que cualquier otra aproximación dada. Estos investigadores indican que la coordinación de dos cantidades variables no es trivial para los estudiantes.

Del mismo modo, Zandieh (2000) considera que la comprensión de la función derivada se apoya en la identificación de la covariación de los valores del dominio de la función derivada con los valores de la tasa de variación de la función original. El razonamiento covariacional también es importante para entender las relaciones entre las integrales definidas e indefinidas (primitivas) y para comprender el Teorema Fundamental del Cálculo. Pero Thompson y Silverman (2008) reconocen que el paso de la integral definida a la función integral supone un salto cualitativo importante: 
[...] una integral definida es a una función integral como 4 es a $\mathrm{x}^{2}$. No se puede enseñar la idea de función si los estudiantes han calculado los valores sólo de una. De forma similar, no pensaríamos que podríamos enseñar la idea de función integral habiendo calculado solo integrales definidas específicas (THOMPSON; SILVERMAN, 2008, p. 46).

Kouropatov y Dreyfus (2014), proponen introducir el concepto de función integral a estudiantes de bachillerato en cuatro etapas, apoyándose en la idea de acumulación. En la primera etapa se espera que los estudiantes hagan aproximaciones de forma intuitiva en el contexto de objetos geométricos:

[...] en la etapa 2 se espera que los alumnos construyan conocimiento sobre la integral definida (es decir, el valor de acumulación), en la etapa 3 de la integral definida con límite superior variable (es decir, la función de acumulación), y en la etapa 4 del Teorema Fundamental del Cálculo [TFC] (es decir, la tasa de variación instantánea de la función de acumulación) (KOUROPATOV; DREYFUS, 2014, p. 537).

La tercera etapa, la construcción de la función integral, la entienden como "un proceso de cambio y cómo la objetivación de este proceso puede ser representado por una gráfica, o expresado por sus propiedades" (KOUROPATOV; DREYFUS, 2014, p. 543). Por ello, estos autores sostienen que los estudiantes tienen que conocer, aunque sea de forma intuitiva:

Que un cambio del punto extremo superior de la acumulación provoca dos cambios:

- un cambio en el valor de la función dada,

- un cambio en el intervalo en el que la función dada se acumula.

Ambos cambios provocan un cambio del valor de acumulación.

- Cómo el cambio del valor de acumulación depende de las propiedades de la función dada (KOUROPATOV; DREYFUS, 2014, p. 544).

Por tanto, ellos consideran que para comprender la función integral $F(x)=\int_{a}^{x} f(t) d t$ es necesario considerar, simultáneamente, tres objetos matemáticos:

$$
x, f(t) \text { y } F(x), \text { siendo } x \in[a, b] \text { y } t \in[a, x]
$$

y comprender cómo varía $f$ en función de $t$ y cómo varía $F$ en función de $x$, es decir, la variación de la integral definida en el intervalo $[a, x]$ cuando $x$ recorre el intervalo $[a, b]$. Esto implica considerar cómo cambia cada uno de los objetos implicados en relación a los otros dos, lo que estos autores denominan razonamiento covariacional complejo. Cuando solo se ponen en relación dos objetos se trata de un razonamiento covariacional simple. Kouropatov y Dreyfus (2014, p. 547) obtuvieron evidencias de "la construcción (a veces en parte) y la consolidación parcial de los conceptos de aproximación, valor de acumulación y función de acumulación". Estos conceptos se consideran anidados: la idea de aproximación anidada en el valor de acumulación (integral definida), y éste en el de función de acumulación (función integral).

Por otra parte, para favorecer la comprensión conceptual de los conceptos del Cálculo 
más allá de la manipulación simbólica, y cómo varían unos objetos en relación a otros, se sugiere utilizar ambientes de aprendizaje donde los estudiantes usen, simultáneamente, distintos tipos de representaciones con el apoyo de la tecnología (FERRARA; PRATT; ROBUTTI, 2006; SWIDAM; YERUSHALMY, 2014). Berry y Nyman (2003) investigaron cómo los estudiantes construían la relación entre el gráfico de la derivada de una función y el de la función. Sus resultados indican que, al comienzo de la actividad, los estudiantes mostraban una visión algebraica del Cálculo y tuvieron dificultad para relacionar la gráfica de una función derivada y la función original, pero el uso de la tecnología permitió desarrollar una mejor comprensión debido a las posibilidades de dinamismo e interacción de y entre las representaciones. En el mismo sentido, Camacho, Depool y Santos-Trigo (2010) mostraron que la utilización de programas de cálculo simbólico permite un cierto progreso en el uso de aspectos gráficos y numéricos del concepto de integral definida.

El estudio que aquí presentamos forma parte de una investigación más amplia sobre la construcción del concepto de integral definida. En este informe, nos centramos en la construcción de la función integral por estudiantes de 16-18 años. Nuestro objetivo es: caracterizar cómo estudiantes de Bachillerato (17-18 años) construyen el concepto de función integral en un experimento de enseñanza utilizando applets.

\section{Marco teórico}

Para analizar cómo los estudiantes de Bachillerato (16-18 años) construyen el significado de función integral, usamos el mecanismo reflexión sobre la relación actividadefecto. Este mecanismo es una elaboración de Simon et al. (2004) a partir de la idea de abstracción reflexiva de Piaget (2001).

Piaget caracterizó tres tipos de abstracción: empírica, pseudoempírica y reflexiva; la abstracción reflexiva se apoya en las dos primeras, pues tiene lugar al abstraer propiedades comunes de varios objetos y realizar acciones sobre ellos, a través de la interiorización y coordinación de las acciones y la creación de nuevos objetos (DUBINSKY, 1991). Esto indica que existe un cambio en la manera en que los estudiantes consideran las estructuras matemáticas. Piaget (2001) entiende que la abstracción es reflexiva en dos sentidos complementarios:

Primero se traspone a un plano superior lo que se toma de un plano inferior (por ejemplo, al conceptualizar una acción). Llamamos a dicha transferencia o proyección un réfléchissement. Segundo, se debe por tanto reconstruir en el nuevo nivel $\mathrm{B}$ lo que se ha tomado del nivel previo A, o establecer una relación entre los elementos extraídos de A y aquellos todavía situados en B. Esta reorganización que 
es forzada por la proyección será llamada reflexión en el sentido estricto (PIAGET, 2001, p. 30).

Esta descripción da lugar a la consideración de dos etapas diferenciadas dentro del proceso de abstracción reflexiva: la etapa de proyección y la etapa de reflexión. En la etapa de proyección ciertas características de las actividades cognitivas de un sujeto (esquemas, acciones, operaciones, estructuras cognitivas...) se separan y se usan con otros fines, es decir, se producen nuevas adaptaciones; en la etapa de reflexión se produce una reorganización de las relaciones entre las acciones, de manera que hay un cambio en las estructuras de conocimiento previas.

El mecanismo reflexión sobre la relación actividad-efecto (SIMON et al., 2004) trata de describir la construcción de un nuevo concepto, intentando operativizar la transposición a un plano superior y la reorganización a las que hace referencia Piaget para explicar el proceso de abstracción. El proceso comienza cuando el estudiante trata de resolver una determinada tarea; la demanda de resolución genera un objetivo en el estudiante que determina una serie de acciones mentales que dependen de sus conocimientos previos. Para poder alcanzar el objetivo, el estudiante realiza una actividad, o secuencia de actividades, prestando atención a los efectos de la actividad realizada.

En el proceso de observación de los efectos de la actividad, el estudiante crea registros mentales de la relación actividad-efecto. En función del efecto obtenido y de la necesidad de alcanzar su objetivo, el estudiante realiza ajustes de manera intencionada para llegar a su objetivo. Al llevar a cabo una nueva actividad o un ajuste de la actividad inicial, ésta produce un nuevo efecto y el estudiante clasifica y compara los registros mentales que relacionan cada actividad con el efecto producido. El término reflexión que da nombre al mecanismo hace referencia a la continua comparación de la relación entre la actividad y su efecto, con relación al objetivo a alcanzar. Esta comparación de registros mentales lleva a la identificación de estructuras y/o patrones en la relación entre actividad y efecto. De esta forma la reflexión lleva al estudiante a la abstracción reflexiva de regularidades en la relación actividad-efecto.

Tzur y Simon (2004) han identificado dos fases en la elaboración de un nuevo concepto: la fase de participación, entendida como el proceso donde el alumno abstrae una regularidad en la relación entre la actividad realizada y el efecto producido, y la fase de anticipación que se refiere al uso de la regularidad abstraída en situaciones distintas a las que se llevó a cabo la abstracción. Roig (ROIG, 2008; ROIG; LLINARES; PENALVA, 2012) ha identificado tres momentos en la fase de participación: proyección, reflexión y anticipación local. En el momento de proyección los alumnos construyen un conjunto de registros o 
unidades de experiencia, en el momento de reflexión abstraen una regularidad a partir de la información procedente del conjunto de registros, y por último, en el momento de anticipación local, aplican la regularidad identificada (la concepción matemática que organiza la situación) a nuevos casos particulares. Roig (2008, p.228) considera, en términos del mecanismo reflexión sobre la relación actividad-efecto, que "las acciones propias de la proyección están anidadas en la coordinación de información que caracteriza la reflexión”, pues se produce en forma paralela a la generación de casos particulares. Este proceso no es, necesariamente, lineal, pues la construcción de un concepto es un mecanismo complejo en el que se dan avances y retrocesos.

Para dar cuenta de este proceso de construcción del conocimiento, se pueden implementar experimentos de enseñanza (GRAVEMEIJER, 2004) que contemplan Ciclos de Enseñanza de las Matemáticas (SIMON, 1995). El diseño e implementación de experimentos de enseñanza tiene en cuenta: el conocimiento del profesor, las trayectorias hipotéticas de aprendizaje y la evaluación del conocimiento de los estudiantes (que proporciona nuevo conocimiento al profesor y cierra un ciclo de enseñanza).

Para generar una trayectoria hipotética de aprendizaje es necesario conocer la comprensión previa de los estudiantes y definir los objetivos de aprendizaje, las tareas matemáticas y las hipótesis sobre cómo se da el proceso de aprendizaje en el contexto de un conjunto particular de tareas. Estos dos últimos puntos son interdependientes y ahí entra en juego la manera en que se caracteriza el mecanismo reflexión sobre la relación actividadefecto, porque se plantea la necesidad de seleccionar aquellas tareas que, desde el conocimiento disponible para los alumnos, sean la base del aprendizaje pretendido (SIMON; TZUR, 2004).

Para generar una trayectoria de aprendizaje que fundamente el experimento de enseñanza se ha considerado el papel que juega el razonamiento covariacional en la construcción del concepto de función integral, teniendo en cuenta los tres objetos matemáticos identificados por Kouropatov y Dreyfus (2014) para comprender la función integral $F(x)=$ $\int_{a}^{x} f(t) d t:$

$$
x, f(t) \text { y } F(x) \text {, siendo } x \in[a, b] \text { y } t \in[a, x]
$$

Estos tres objetos matemáticos deben considerarse teniendo en cuenta cómo varía $f$ en función de $t$ en el intervalo $[a, x]$ y $F$ en función de $x$ en el intervalo $[a, b]$, así como la dirección del cambio y la cantidad de cambio.

Para ayudar a los estudiantes a identificar estos objetos matemáticos y la coordinación 
entre sus variaciones se propone el uso de la tecnología como instrumento de mediación semiótica (FERRARA; PRATT; ROBUTTI, 2006), utilizando representaciones de tipo numérico, gráfico y algebraico. Estas representaciones no son meras ilustraciones de los conceptos, pues la permite actuar sobre ellas. Entre estas tecnologías se encuentra el uso de applets diseñados ad hoc, que se manejan con facilidad y permiten visualizar la superficie bajo una curva en un intervalo, calcular el valor de la integral definida $\int_{a}^{x} f(t) d t$ to visualizar la función integral $F(x)$. En estos applets, al mover uno de los extremos del intervalo aparecen los valores de la integral definida y se puede representar de forma dinámica la función integral de una función moviéndose sobre el gráfico de la función; la gráfica de la función integral se construye, así, de forma dinámica, lo que permite constatar la relación entre una función y su función integral; al mismo tiempo pueden aparecer en pantalla las coordenadas del punto que se mueve sobre el gráfico de la función y la expresión analítica de la función y de su función integral.

En este contexto nuestra pregunta de investigación es: ¿qué características de la construcción de la función integral, en el marco del mecanismo reflexión sobre la relación actividad-efecto, es posible identificar a partir de un experimento de enseñanza que integra applets?

\section{Método}

\subsection{Participantes y contexto}

Los participantes en esta investigación fueron 15 estudiantes de $2^{\circ}$ de Bachillerato (1718 años) de la modalidad de Ciencias de la Naturaleza y de la Salud. Se formaron 6 parejas y un trío agrupados por similar rendimiento académico. Estos alumnos habían estudiado en el curso anterior el límite de una función en un punto con un enfoque procedimental, y previamente a su participación en el experimento de enseñanza que aquí se presenta, habían estudiado el concepto de derivada con un enfoque conceptual usando applets diseñados ad hoc con Geogebra por la primera autora de este trabajo. El papel de la profesora era aclarar dudas y moderar una puesta en común en cada sesión.

El experimento de enseñanza plantea el estudio de la integral definida a partir del cálculo del área de una superficie bajo una curva (TURÉGANO, 1998) y se articula de la siguiente manera: 
1. Cálculo del área del círculo por el método de agotamiento.

2. Aproximación del área de superficies bajo una curva mediante suma de áreas de rectángulos.

3. Diferencia entre área bajo una curva en un intervalo e integral definida de la función definida por la curva en dicho intervalo: definición de la integral definida.

4. Propiedades de la integral.

5. Introducción de la función integral, del teorema fundamental del Cálculo y la regla de Barrow.

Los estudiantes podían apoyarse en applets diseñados ad hoc con Geogebra por la primera autora de este artículo, con las características antes señaladas. En este trabajo nos centramos en la introducción de la función integral para identificar las características del proceso de construcción del concepto. Estas características, en relación a los diferentes momentos de la fase de participación, se presentan mediante dos estudios de casos.

\subsection{Trayectoria hipotética de aprendizaje de la construcción del concepto de función integral}

La Figura 1 muestra la trayectoria hipotética de aprendizaje de la construcción de la función integral que articuló el experimento de enseñanza, en relación a los momentos de la fase de participación. Nuestra hipótesis es que los estudiantes, tras familiarizarse con un applet dando valores a los parámetros $m$, pendiente, y $n$, ordenada en el origen de una recta de ecuación $y=m x+n$, y calcular el área bajo la recta en un intervalo fijo $[a, t]$ donde la función es positiva, experimentarán variando el extremo $t$ del intervalo en casos particulares (con funciones constantes, lineales y afines), para obtener la función que expresa el valor del área bajo la recta. Esto les permitirá relacionar una función constante, lineal o afín, y el área bajo la recta en un intervalo, coordinar la variación de tres cantidades (extremo $t$ superior del intervalo, valor de la función en dicho punto y valor del área bajo la recta en el intervalo [0, t]). La coordinación de las representaciones geométricas (gráficas de las rectas y de la función integral) y algebraicas de una función y su función integral (ecuaciones de las rectas y de su función integral) les debe llevar a inferir que la función integral de una función representada por una recta es una función polinómica de grado 1 o 2, lo que les puede situar en posición de aplicar las regularidades observadas a nuevas situaciones (extender).

Se propusieron dos tareas con el objetivo de que los estudiantes generasen un conjunto de registros de experiencia de la relación entre una función y su función integral. El objetivo 
de la primera tarea (Función Integral I; Figura 2) era que los estudiantes llegaran a expresar la relación entre una función constante, lineal o afín, y su función integral. Para ello disponían de una guía de trabajo y se podían apoyar en un applet (Figura 3) donde podían modificar los parámetros $m$ y $n$ de una recta de ecuación $y=m x+n$ mediante deslizadores, y los extremos de un intervalo $[a, t]$, lo que les permitía visualizar los polígonos cuya área debían calcular. Para responder a esta tarea los estudiantes necesitaban identificar tres objetos matemáticos $(t, f(x)$ $=m x+n$ donde $x \in[a, t]$, y la función que expresa el valor del área del polígono en función de t), ya fuese de manera gráfica, numérica o algebraica, y cómo varían unos en relación a otros.

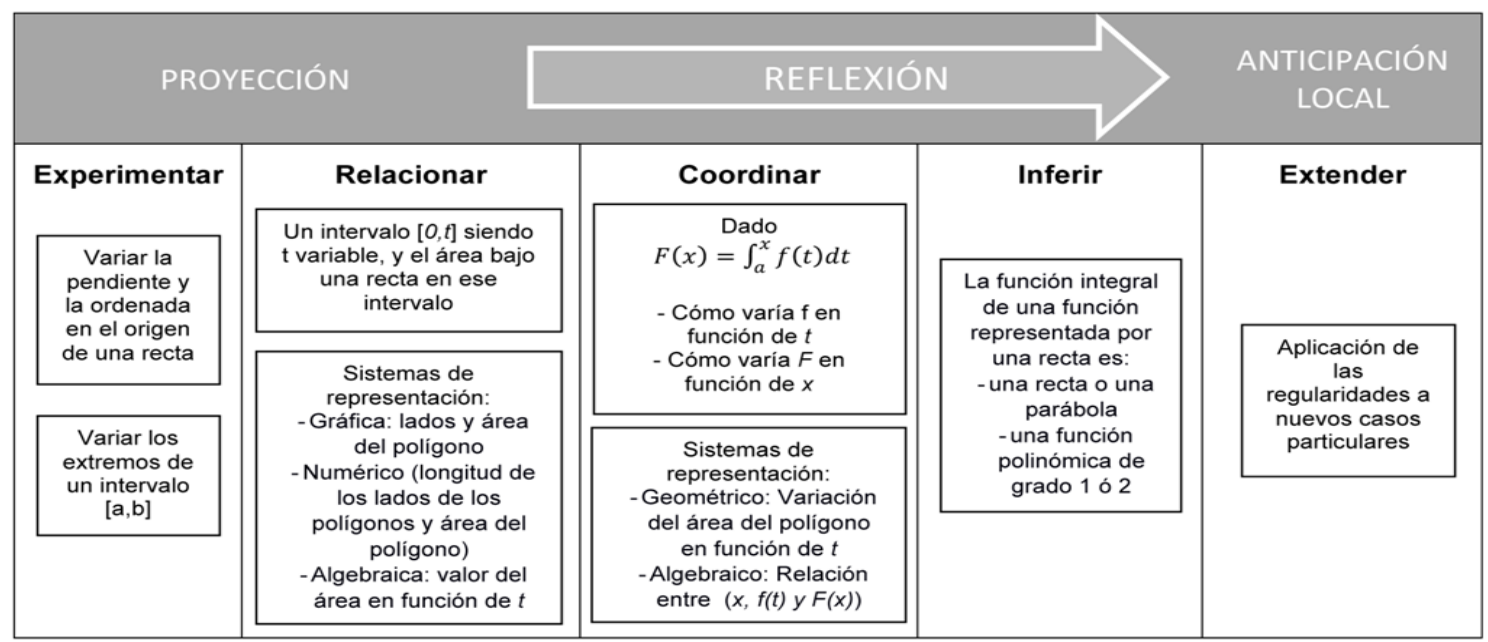

Figura 1 - Trayectoria hipotética de aprendizaje para la construcción de la función integral, basada en los momentos de la fase de participación en el proceso de abstracción reflexiva

Fuente: Aranda (2015)

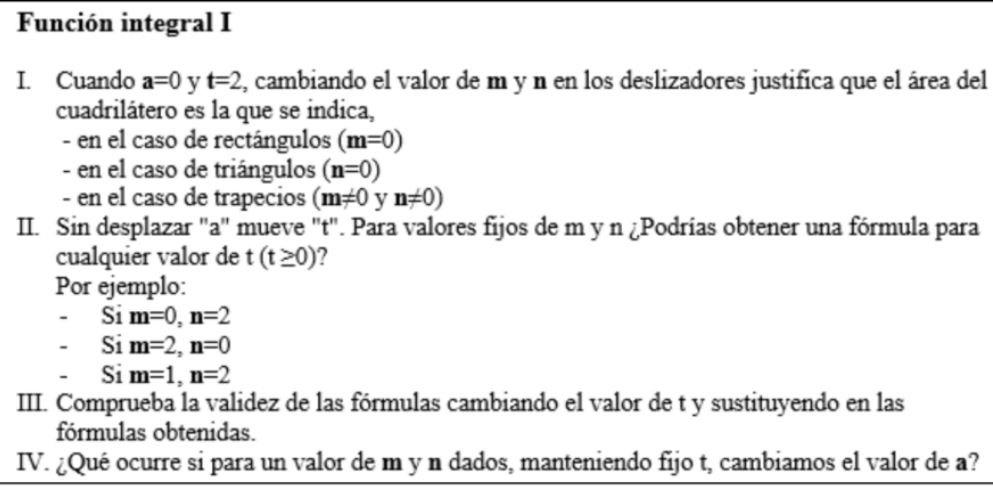

I. Cuando $\mathbf{a}=0 \mathrm{y} \mathbf{t}=2$, cambiando el valor de $\mathbf{m}$ y $\mathbf{n}$ en los deslizadores justifica que el área del cuadrilátero es la que se indica,

- en el caso de rectángulos $(\mathbf{m}=0)$

- en el caso de triángulos $(\mathbf{n}=0)$

- en el caso de trapecios $(\mathbf{m} \neq 0$ y $\mathbf{n} \neq 0)$

II. Sin desplazar "a" mueve " $t$ ". Para valores fijos de $\mathrm{m}$ y $\mathrm{n}$ ¿Podrias obtener una fórmula para cualquier valor de $t(t \geq 0)$ ?

Por ejemplo:

Si $\mathbf{m}=0, \mathbf{n}=2$

Si $\mathbf{m}=2, \mathbf{n}=0$

Si $\mathbf{m}=1, \mathbf{n}=2$

III. Comprueba la validez de las fórmulas cambiando el valor de $t \mathrm{y}$ sustituyendo en las fórmulas obtenidas.

IV. ¿Qué ocurre si para un valor de $\mathbf{m}$ y $\mathbf{n}$ dados, manteniendo fijo $t$, cambiamos el valor de a?

Figura 2 - Guía de trabajo de la tarea Función Integral I Fuente: Aranda (2015)

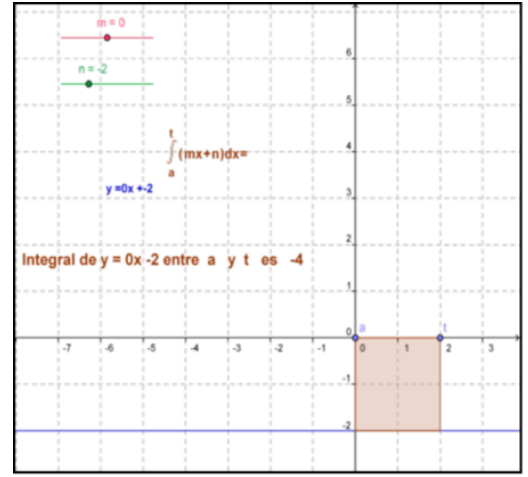

Figura 3 - Applet de apoyo de la tarea Función Integral I Fuente: Aranda (2015)

El objetivo de la segunda tarea (Función Integral II; Figura 4) era relacionar la gráfica de una función y la de su función integral; en ella se pedía dibujar las gráficas de las funciones que representan el área bajo una recta en un intervalo donde la función es positiva. Los estudiantes podían verificar el resultado con ayuda de un applet. En este applet los estudiantes podían modificar los parámetros $m$ y $n$ de una recta de ecuación $y=m x+n$ 
mediante deslizadores, para representar la función de la que se quería obtener su función integral. Además, al desplazar el extremo superior del intervalo $[a, x]$, aparecía representada gráficamente $F(x)$ en este intervalo, que es la función integral de la recta $y=m x+n$. Se indicaba que el valor que toma la función $\mathrm{F}$ en un punto $x$ es el valor del área bajo la recta en el intervalo $[a, x]$ (Figura 5)

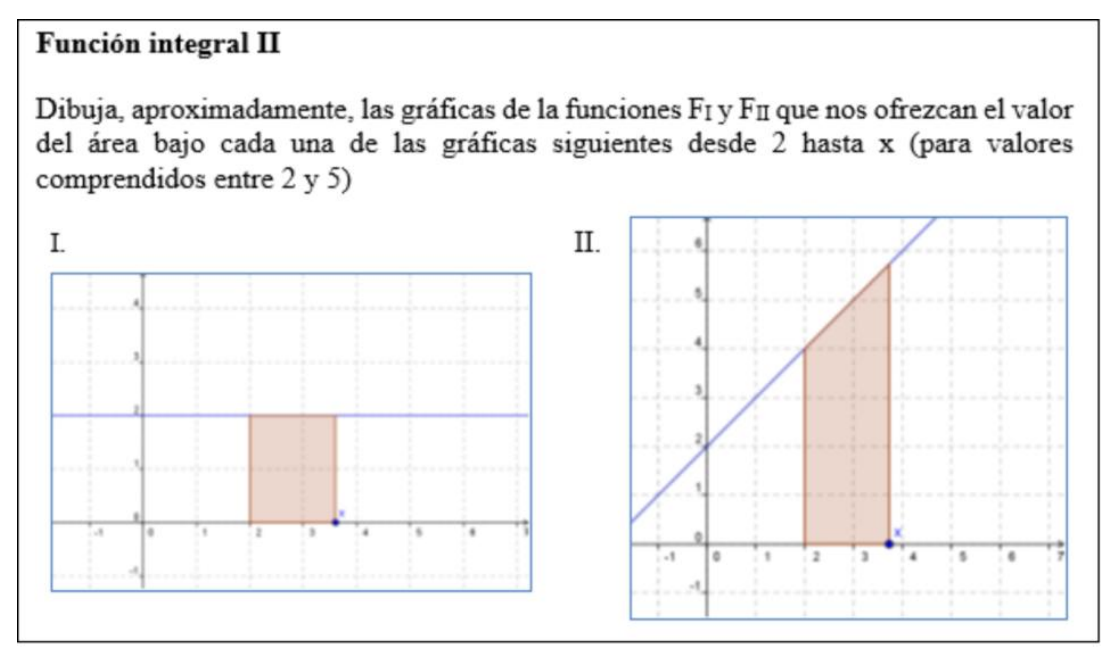

Figura 4 - Tarea Función Integral II Fuente: Aranda (2015)

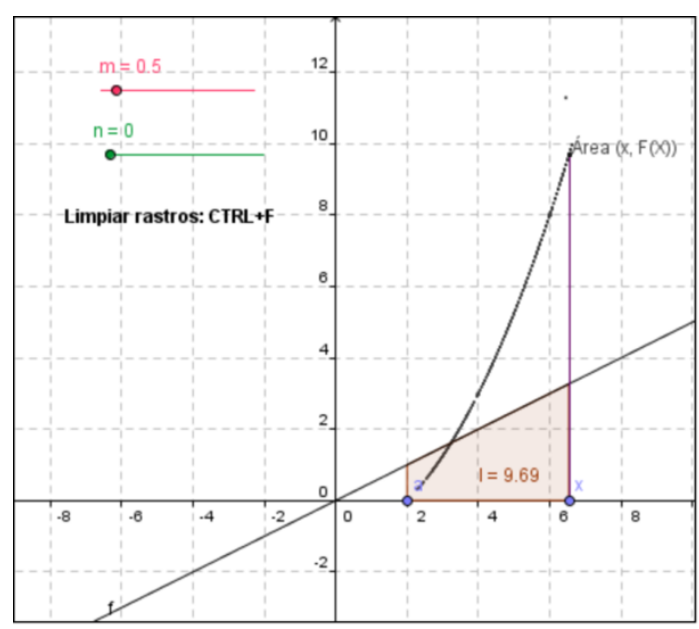

Figura 5 - Apple para comprobar los resultados obtenidos en Función integral II Fuente: Aranda (2015)

\subsection{Recogida y análisis de datos}

Los datos son: (1) las acciones realizadas por los estudiantes con los applets en la resolución de las dos tareas; (2) las declaraciones orales de las parejas o trío; y (3) las hojas de respuesta. Los dos primeros tipos de datos se recogieron con el programa CamStudio (C) que registra simultáneamente en archivos digitales la actividad en la pantalla del ordenador (vídeo) y lo que se dice mientras se trabaja ante el ordenador (audio). 
Para analizar los diferentes datos se tuvo en cuenta la trayectoria hipotética de aprendizaje de la construcción de la función integral, en concreto los momentos de la fase de participación en la construcción de este concepto (proyección, reflexión o anticipación local) y las acciones en cada una de esas fases (experimentar y relacionar en el momento de proyección; coordinar e inferir en el de reflexión; y, por último, extender en el de anticipación local). Estas momentos y estas acciones se han especificado en la Figura 1, teniendo en cuenta el mecanismo reflexión sobre la relación actividad-efecto (SIMON et al., 2004) en la construcción del significado de un nuevo concepto, las fases y momentos en esta construcción (ROIG; LLINARES; PENALVA, 2012; TZUR; SIMON, 2004) y la idea de trayectoria hipotética de aprendizaje (SIMON, 1995).

Primero, se transcribieron las comunicaciones orales y las acciones realizadas con los applets. Se consideró como unidad de análisis las acciones o declaraciones - orales o escritas de los estudiantes. Cada unidad de análisis se codificó, teniendo en cuenta las acciones de experimentar, relacionar, inferir, coordinar y extender consideradas en la trayectoria hipotética de aprendizaje, y los distintos momentos de la fase de participación en el proceso de abstracción reflexiva (Figura 1). Dos investigadores codificaron por separado las acciones y momentos identificados en las transcripciones. Las codificaciones realizadas fueron discutidas y las discrepancias analizadas. Por último, se describieron las trayectorias de aprendizaje a través de las acciones realizadas y los distintos momentos de la fase de participación, agrupándose las parejas de estudiantes dependiendo del momento de la fase de participación en que se encontraban.

En la sección de resultados describimos las características identificadas en la manera en la que los estudiantes construían el concepto de función integral.

\section{Resultados}

Presentamos diferentes características del proceso de construcción a través de dos estudios de un grupo de tres estudiantes y una pareja, que se encuentran en distintos momentos de la fase de participación:

Caso 1: Sin evidencias de relacionar una función y su función integral (momento de proyección) (trío $\mathrm{V}-\mathrm{AV}-\mathrm{AJ})$.

Caso 2: Con indicios de razonamiento covariacional complejo: coordinación de la variación de tres cantidades en casos particulares (momento de reflexión) (pareja L-M) 


\subsection{Caso 1}

Los estudiantes del caso 1 (trío V-AV-AJ) se encuentran en el momento de proyección pues experimentaron variando los parámetros de la recta y los extremos del intervalo, y calcularon el área bajo una recta en un intervalo fijo, pero no fueron capaces de relacionar como varían los valores de la función (el área del polígono) en relación al extremo $t$ variable del intervalo $[a, t]$. Además, estos estudiantes tenían dificultad para manejar el lenguaje analítico. Emplearon mucho tiempo para resolver la primera tarea y no les dio tiempo a responder a la segunda.

En la tarea Función integral I estos estudiantes visualizaron los polígonos con el applet (rectángulos, triángulos y trapecios) y no tuvieron dificultades para expresar el área del rectángulo en función de $n$ (ordenada en el origen de la recta) y $t$ (Figura 6).

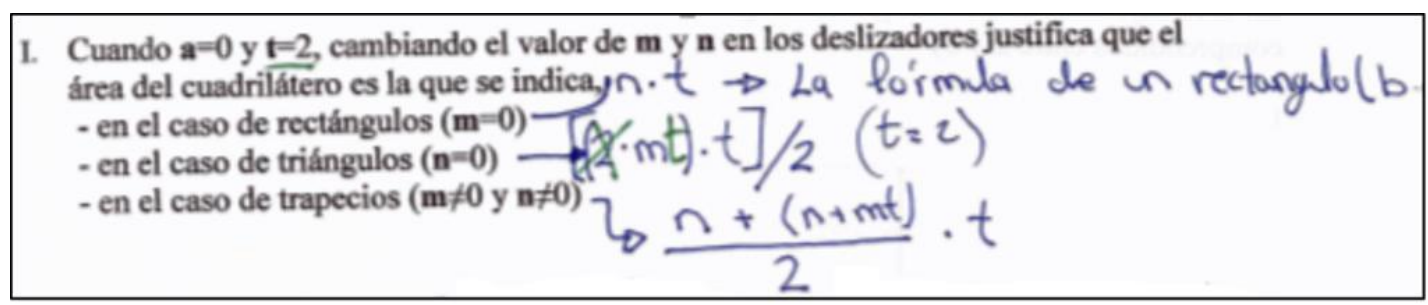

Figura 6 - Respuesta del trío V-AV-AJ a la primera cuestión de Función Integral I Fuente: Aranda (2015)

En cambio, en el caso de los triángulos $(n=0$, función $y=3 x)$ encontraron dificultad para expresar el área porque daban a $t$ el valor 2 para calcular la altura, sin embargo consideraban que la base era la variable $t$ en lugar del valor 2, como se puede observar en un intercambio entre V y AV (Figura 7), en particular el intercambio [148]. 


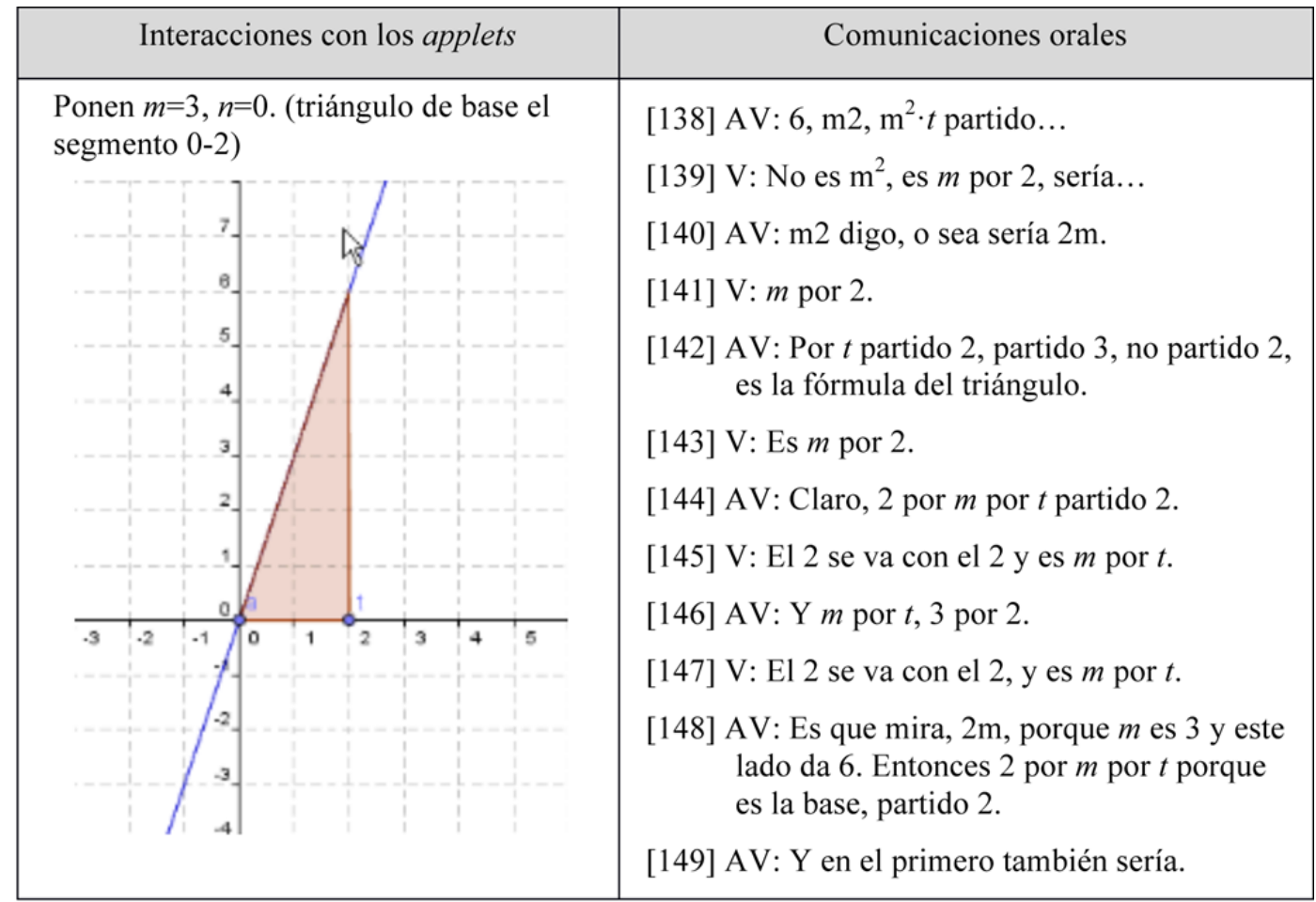

Figura 7 - Intercambio entre V y AV para calcular el área de un triángulo Fuente: Aranda (2015)

La profesora les hizo notar la forma en que estaban considerando $t$, y a continuación usaron $t$ como variable tanto para la base como para la altura del triángulo. Con esta ayuda llegaron a expresar el área del triángulo en función de $t$ como $m t \cdot t / 2$ (Figura 6). Asimismo, expresaron las demás áreas mediante fórmulas, en función de los parámetros $m$ y $n$ de una recta de ecuación $y=m x+n$ y del extremo $t$ del intervalo, siendo $t$ fijo (Figura 6).

Cuando se le pidió la expresión del área para cualquier valor de $t$ (cuestión II en tarea 1, Figura 2), escribieron las fórmulas que expresan las áreas de los polígonos e interpretaron cada una de ellas como un objeto matemático donde hay que dar valores a las variables, como se muestra en el diálogo, donde estos estudiantes dan a $t$ los valores 7 y 3 (Figuras 8 y 9), y no como una función que relaciona el extremo $t$ superior del intervalo y el valor del área bajo la recta en el intervalo $[a, t]$ en función de $t$,

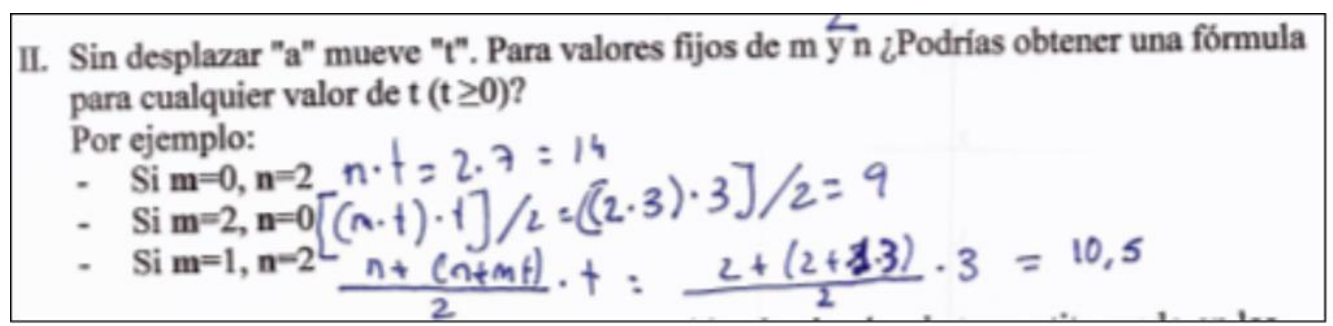

Figura 8 - Respuesta del trío V-AV-AJ a la segunda cuestion de Función Integral I Fuente: Aranda (2015) 


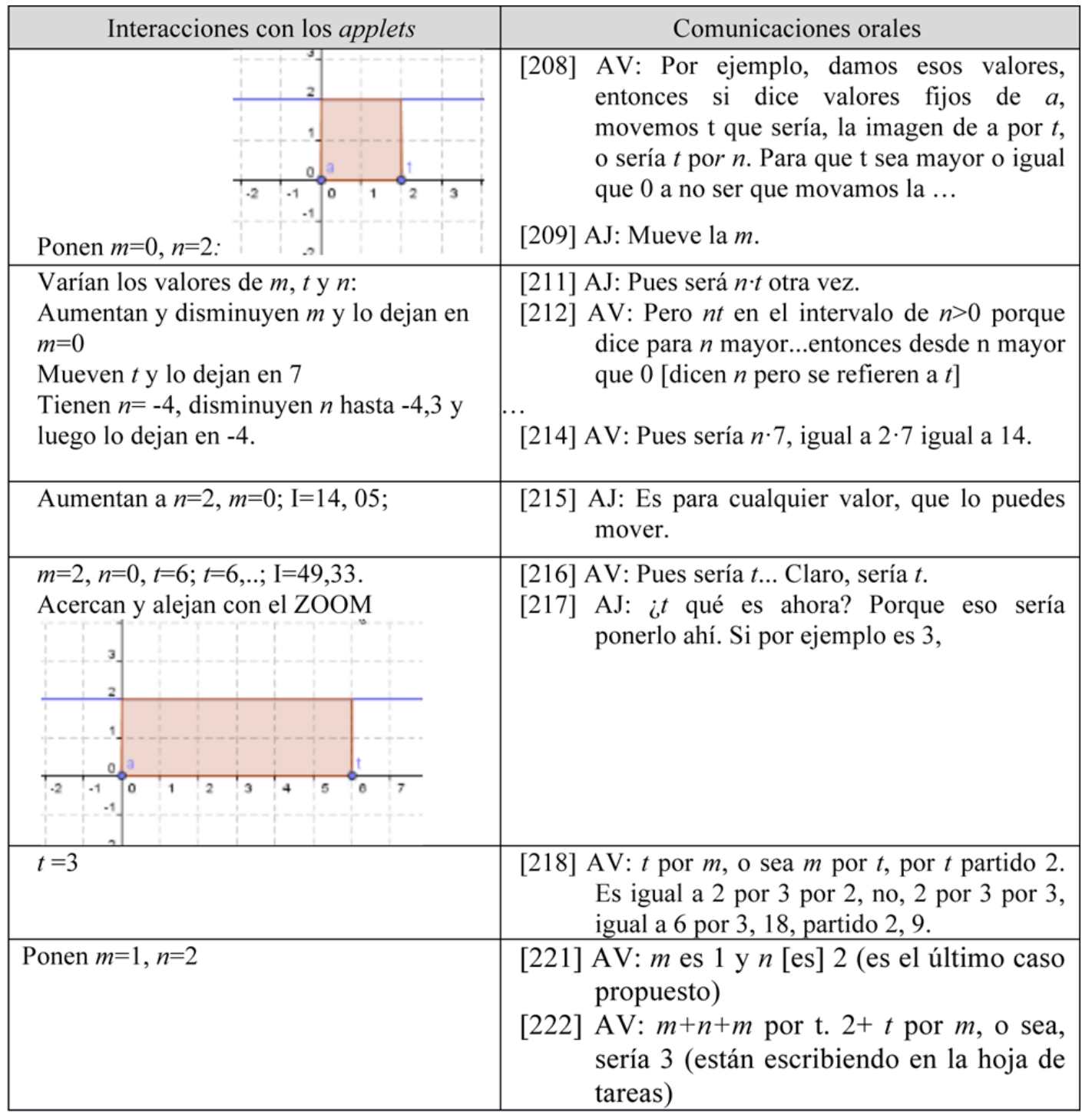

Figura 9 - Diálogo de la pareja V-AV resolviendo la cuestión II de la tarea Función integral I Fuente: Aranda (2015)

Posteriormente, comprobaron la validez de las fórmulas en casos particulares usando el applet, que manejaron sin dificultad (cuestión III). Estos estudiantes fueron capaces de identificar la fórmula del área de una superficie bajo una recta en un intervalo $[0, t]$, pero no de atender a la coordinación de dos cantidades variables: el extremo $t$ variable del intervalo y el valor del área bajo la recta en el intervalo $[0, t]$, por ello consideramos que se sitúan en el momento de proyección.

\subsection{Caso 2}

Los estudiantes del caso 2 (L-M) relacionaron una función constante, lineal o afín, y la función integral, y expresaron esta relación gráfica y/o analíticamente. La forma en que esta pareja expresó el área de las superficies bajo las rectas en función de $t$ sin darle un valor 
particular (Figura 10), es una evidencia de que establecía una relación funcional entre el extremo $t$ variable de un intervalo, la función $f(x)=m x+$ n y el área bajo la recta en el intervalo $[0, t]$; además la expresaron analíticamente en función de la variable $t$, y de los parámetros $m$ y $n$. Este comportamiento podemos considerarlo como propio del momento de reflexión.

I. Cuando $\mathbf{a}=0$ y $\mathbf{t}=2$, cambiando el valor de $\mathbf{m}$ y $\mathbf{n}$ en los deslizadores justifica que el

$$
\begin{aligned}
& \text { área del cuadrilátero es la que se indica, } \\
& \text { - en el caso de rectángulos }(m=0) \quad A=e \cdot l=2 \cdot(-2)=-4 \\
& \text { - en el caso de triángulos }(n=0) \quad A=\frac{b \cdot h}{2}=\frac{t \cdot n}{2} \\
& \text { - en el caso de trapecios }(m \neq 0 \text { y } \mathbf{n} \neq 0) \\
& \quad A=\frac{B+b}{2} \cdot h=\frac{n+n+m t}{2} \cdot t=\frac{2 n+m t}{2} \cdot t
\end{aligned}
$$

II. Sin desplazar "a" mueve " $t$ ". Para valores fijos de $m$ y n ¿Podrías obtener una fórmula para cualquier valor de $t(t \geq 0)$ ?

Por ejemplo:

- $\quad$ Si $\mathbf{m}=0, \mathbf{n}=2$

- $\quad$ Si $\mathbf{m}=2, \mathbf{n}=0$

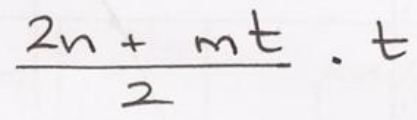

- $\quad$ Si $\mathbf{m}=1, \mathbf{n}=2$

III. Comprueba la validez de las fórmulas cambiando el valor de t y sustituyendo en las fórmulas obtenidas.

\section{Comprobado.}

Figura 10 - Respuesta L-M a la tarea Función Integral I

Fuente: Aranda (2015)

En segundo lugar, esta pareja de estudiantes fue capaz de representar gráficamente la función integral de una función constante y afín (tarea Función Integral II). Cuando se le pidió que representara gráficamente la función que expresa el valor del área bajo la gráfica de una función constante en el intervalo $[2, x]$, comenzaron apoyándose en un resultado obtenido anteriormente (el área bajo una recta de ecuación $f(x)=m x+n y=m x+n$ en el intervalo [0, t] es $(2 \mathrm{n}+\mathrm{mt}) \mathrm{t} / 2)$, y sustituyeron los parámetros $n$ y $m$ por sus valores $(n=2, m=0)$ y $t$ por $x$; así obtuvieron $f(x)=2 x$, pero comprobaron, dando valores, que la expresión no era correcta debido a que el extremo izquierdo no era 0 - y tantearon cuál debería ser la expresión analítica, como muestra el siguiente diálogo:

[255] L: 4-2, sería $x-2,2$ por $x-2$

[256] M: ¿Por qué es menos?

[257] L: Pero es que si la x fuera 4, el área de esto sería 4-2, 2, por 2 [se están refiriendo a la base, 4-2, por altura, 2], 4.

[258] M: Ah, es que pone desde 2, entonces si pone desde 2, empezaría aquí.

[259] L: $x$-2.

[260] M: Claro, menos 2. Pero por menos 2 detrás, 2 por -2.

[261] L: ¿Pero no es 2 por $x$-2? Es que no es lo mismo $2 x$ - 2 que 2 por $x$-2. (ARANDA, 2015, p. 135)

Y siguieron, discutiendo cuál era la forma correcta, dando valores a $x$ : 
[274] M: Entonces llegaría aquí. En 2 sería cero, en 3 habría subido al 2 ¿no? En 3 habría subido hasta aquí y sería 2 por 1.

[275] L. ¿La tengo que dibujar?

[276] M: Sí, tiene que pasar de ahí y tiene que llegar hasta ahí. (ARANDA, 2015, p. 135)

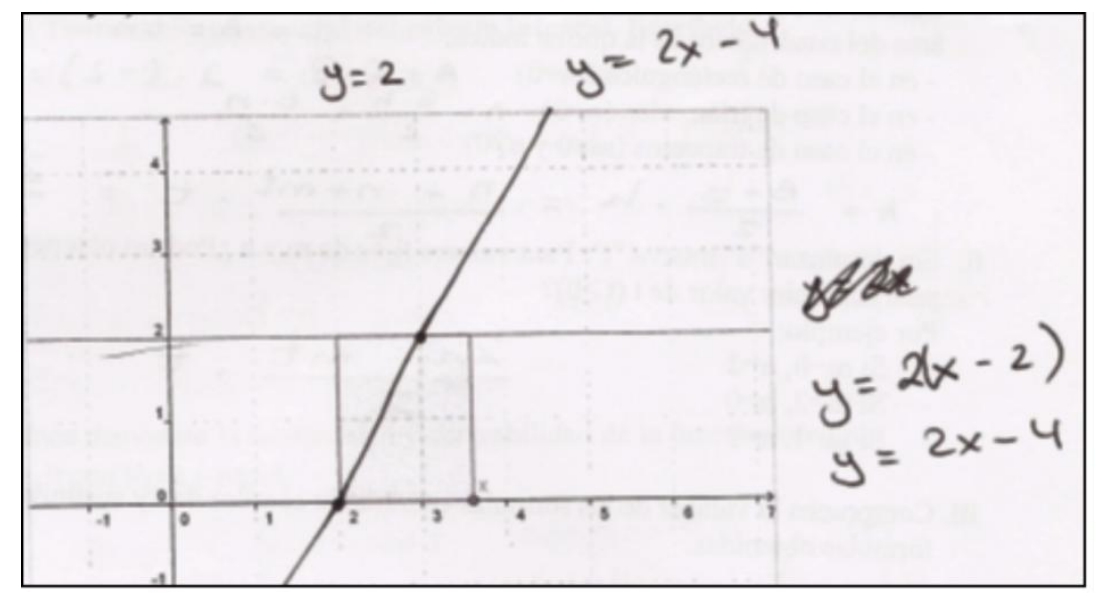

Figura 11 - Respuesta de la pareja L-M a la tarea Función Integral II (función constante) Fuente: Aranda (2015)

Estos estudiantes manejaron, en este caso, distintas representaciones de la función integral: algebraica (como conjetura y refinamiento de la misma), numérica (dando valores para comprobar la conjetura) y gráfica (como respuesta a la cuestión planteada), lo que les ayudó a dar la respuesta correcta. Por tanto, al establecer la relación entre una función y su función integral, estos estudiantes mostraron evidencias de coordinación de distintas formas de representación de una función para dar cuenta de la relación entre las variaciones de los diferentes objetos matemáticos. Este comportamiento es un indicio de razonamiento covariacional simple, pues establece la coordinación entre dos cantidades variables: el extremo superior $x$ del intervalo y el valor del área bajo la recta $y=2$ en el intervalo $[2, x] \mathrm{y}$ cómo varía el área en función de $x$.

Por otra parte, no hemos encontrado evidencias de que estos estudiantes pudieran inferir que la función integral de una función constante es una función lineal o afín, dependiendo de que el extremo $a$ del intervalo $[a, x]$ sea 0 o distinto de 0 , lo que habría sido una evidencia más de la construcción del concepto de función integral.

Sin embargo, en la cuestión que pedía expresar el área bajo una recta que representa una función afín en el intervalo $[2, x]$, la pareja L-M afirmó que la función que expresa el valor del área bajo una recta "tiene que dar una parábola [...] porque de grado 1 tiene que pasar a grado 2" (PAREJA L-M). Esta afirmación, que luego comprobó dando valores, pero sin hacer referencia explícita al crecimiento de la función o a puntos singulares como el vértice, puede ser considerado otra evidencia de razonamiento covariacional, pues establece una coordinación entre parejas de funciones. Esta manera de proceder pone de manifiesto 
cómo estos estudiantes van construyendo progresivamente el concepto de función integral, evidenciado en el siguiente diálogo donde un miembro de la pareja, M, lleva la iniciativa y el otro, L, le cuestiona:

[322] M: A ver, tiene que dar una parábola.

[323] L: ¿Por qué?

[324] M: Porque de grado 1 tiene que pasar a grado 2.

[325] L: Vale, entonces.

[326] M: Pero vamos a hacerlo, a ver, aquí es cero.

[327] L: ¿Que aquí qué?

[328] M: En ese valor ¿cuánto vale el área?

[329] L: ¿En 2? No entiendo lo que me estás diciendo. A ver, si $x=3$, el área de esto es 9.

(ARANDA, 2015, p. 137)

Estos estudiantes siguieron dando valores y dibujaron la parábola, aproximadamente con algunos errores en el gráfico (Figura 12), pero, sin embargo, no llegaron a expresar analíticamente la ecuación de la parábola.

Estos estudiantes usaron dos tipos de representaciones de la función integral: numérica (dando valores para comprobar la conjetura) y gráfica (representando la parábola que pasa por los puntos), pero no la algebraica. Esto podría deberse a que no se apoyaron en el resultado obtenido en el bloque II de esta tarea, como hicieron en el caso de la función constante. Su comportamiento muestra que han sido capaces de coordinar la dirección del cambio y la cantidad de cambio.

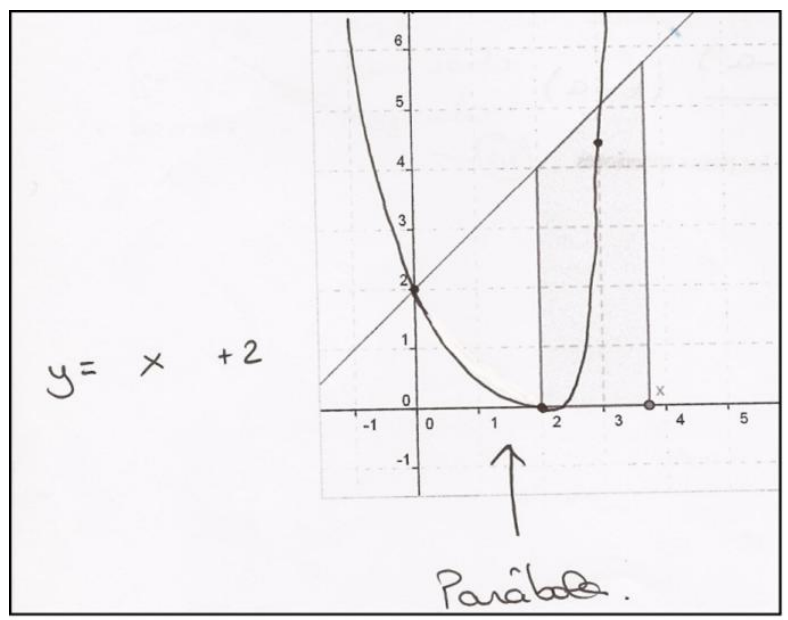

Figura 12- Respuesta de la pareja L-M a la tarea Función Integral II (función afín) Fuente: Aranda (2015)

Podemos decir que esta pareja de estudiantes, a medida que ha ido resolviendo las tareas, ha ido construyendo progresivamente el concepto de función integral, desde un razonamiento covariacional más simple a otro más complejo. En primer lugar, los estudiantes relacionaron el extremo superior $\mathrm{x}$ del intervalo $[a, x]$ con la integral definida de una función $f(t)$ constante, lineal o afín, en dicho intervalo; en segundo lugar, relacionaron esta función $f(t)$ 
y su función integral $F(x)$ para valores positivos de la función, con $x \in[a, b]$ y $t \in[a, x]$; en tercer lugar, relacionan familias de funciones. En este proceso los estudiantes se apoyaron en distintos sistemas de representación: algebraico, gráfico y numérico, para establecer la coordinación entre los diferentes objetos matemáticos. Por ello, podemos considerar que estos estudiantes están en el momento de reflexión de la fase de participación.

\section{Discusión}

En este trabajo nos hemos planteado la siguiente pregunta: ¿qué características de la construcción de la función integral, en el marco del mecanismo reflexión sobre la relación actividad-efecto es posible identificar a partir de un experimento de enseñanza que integra applets?

Hemos identificado dos características que definen dos momentos de la transición de la fase de participación a la de anticipación en el proceso de abstracción reflexiva: los momentos de proyección y reflexión. En el de proyección (caso 1) los estudiantes construyen un conjunto de registros o unidades de experiencia y en el de reflexión abstraen regularidades a partir del conjunto de registros (caso 2).

Los estudiantes del caso 1 se sitúan en un momento inicial de la construcción de la función integral, al construir registros de experiencia identificando la fórmula del área de una superficie bajo una recta en un intervalo $[0, t]$ en el que la función es positiva, que corresponde al valor de la integral definida de la función representada por la recta en dicho intervalo. Pero no mostraron evidencias de abstraer regularidades atendiendo a la coordinación de dos cantidades variables: el extremo $t$ variable del intervalo y el valor del área bajo la recta en dicho intervalo, por ello consideramos que no hay evidencias de razonamiento covariacional y se sitúan en el momento de proyección. En cambio los estudiantes del caso 2, que se sitúan en el momento de reflexión, abstrajeron regularidades poniendo en relación estas dos variables que varían simultáneamente, mostrando evidencias de razonamiento covariacional.

El estudios de casos que hemos realizado muestra que el primer salto cognitivo del momento de proyección al de reflexión se produce cuando los estudiantes son capaces de relacionar el extremo superior del intervalo y el valor de la integral de la función en ese intervalo, donde el extremo inferior es un valor fijo y el extremo superior varía, que es una forma de covariación simple. En estos estudiantes hemos identificado un segundo salto cognitivo que se produce cuando son capaces de ver la covariación compleja (SEALEY, 
2006; THOMPSON; SILVERMAN, 2008) de las tres cantidades que varían simultáneamente y que intervienen en el concepto de función integral, es decir, la variable $x, x \in[a, b]$, una función $f(t), t \in[a, x]$ y la función integral $F(x)=\int_{a}^{x} f(t) d t$, en casos sencillos (en nuestro experimento de enseñanza con funciones constantes, afines y lineales que toman valores positivos en el intervalo).

Nuestros resultados muestran la complejidad del razonamiento covariacional y las dificultades que tienen los estudiantes; sin embargo, indican que el razonamiento covariacional aplicado a los conceptos fundamentales del Cálculo es accesible a estudiantes de bachillerato, como ya lo han puesto de manifiesto otros estudios (CARLSON et al., 2002; CONFREY; SMITH, 1994; JOHNSON, 2015; KOUROPATOV; DREYFUS, 2014; THOMPSON, 1994a). Nuestra investigación aporta nueva información identificando aspectos específicos del razonamiento covariacional que resultan problemáticos para los estudiantes, como la forma en que interpretan una expresión algebraica dando valores a las variables en lugar de establecer una relación funcional (caso 1). También, que los estudiantes son capaces de apoyarse en las fórmulas del área de una superficie o en la gráfica de una función para expresar la variación de la integral definida en función del extremo superior del intervalo y construir así la función integral (caso 2).

Nuestra investigación aporta información para complementar la trayectoria de aprendizaje (las dos características del proceso de construcción del concepto de función integral), y, al mismo tiempo, materiales curriculares (las tareas) que pueden ser útiles a los profesores para identificar y promover el razonamiento covariacional en los estudiantes. Las guías para realizar las tareas del experimento de enseñanza y el manejo de distintas representaciones (numéricas, algebraicas y gráficas), ayudaron a la pareja del caso 2 que se encontraba en el momento de reflexión a identificar la función que representa el área bajo una recta en el intervalo $[a, x]$ para la función constante, lineal y afin, haciendo conjeturas y comprobaciones. En cuanto al uso de la tecnología, - los applets como instrumentos de mediación semiótica - nuestros resultados confirman que el uso de distintos sistemas de representación permite a los estudiantes desarrollar una mejor comprensión de la aproximación gráfica al Cálculo, superando la visión algorítmica y analítica, al mismo tiempo que proporciona un entorno favorable para construir una red de ideas relacionadas (ARANDA; CALLEJO, 2011; BERRY; NYMAN, 2003; HONG; THOMAS, 1997). 


\section{Referencias}

ARANDA, C. Análisis de la construcción del concepto de integral definida en estudiantes de bachillerato. Tesis doctoral (inédita). Universidad de Alicante, 2015.

ARANDA, C.; CALLEJO, M. L. Aproximación al concepto de función primitiva: un experimento de enseñanza con applets de geometría dinámica. In: SIMPOSIO NACIONAL DE LA SOCIEDAD ESPAÑOLA DE INVESTIGACIÓN EN EDUCACIÓN MATEMÁTICA, 15., 2011, Ciudad Real. Proceedings... Ciudad Real: Universidad de Castilla-La Mancha, 2011. p. 247-255.

AZCÁRATE, C. et al. Didáctica del Análisis Matemático: Una revisión de las investigaciones sobre su enseñanza y aprendizaje en el contexto de la SEIEM. Primera edición. ed. La Laguna: Universidad de La Laguna, 2015. 248 p.

BERRY, J. S.; NYMAN, M. A. Promoting students' graphical understanding of the calculus. Journal of Mathematical Behavior, Norwood, v. 22, n. 4, p. 479-495, 2003.

CAMACHO, M.; DEPOOL, R.; SANTOS-TRIGO, M. Students' use of Derive software in comprehending and making sense of definite integral and area concepts. CBMS Issues in Mathematics Education, Providence, v. 16, p. 35-67, 2010.

CARLSON, M. et al. Applying covariational reasoning while modeling dynamic events: A framework and a study. Journal for Research in Mathematics Education, Reston, v. 33, n. 5, p. 352-378, nov. 2002.

CONFREY, J.; SMITH, E. Exponential functions, rates of change, and the multiplication unit. Educational Studies in Mathematics, Dordrecht, v. 26, n. 2-3, p. 135-16, mar. 1994.

COTRILL, J. et al. Understanding the limit concept: Beginning whit a coordinated process scheme. Journal of Mathematical Behavior, Norwood, v. 15, n. 2, p. 167-192, jan. 1996.

DUBINSKY, E. Reflective abstraction in advanced mathematical thinking. In: TALL, D. (Ed.). Advanced Mathematical Thinking. Dordrecht: Ed. Kluwer, 1991. p. 95-126.

FERRARA, F.; PRATT, D.; ROBUTTI, O. The role and uses of technologies for the teaching of algebra and calculus. In: GUTIERREZ, A; BOERO, P. (Ed.). Handbook of Research on the Psychology of Mathematics Education. Past, Present and Future. Rotterdam/Taipei: Ed. Sense Publishers, 2006. p. 237-273.

GONZALEZ-MARTIN, A.; CAMACHO, M. What is first-year mathematics students' actual knowledge about improper integrals? International Journal of Mathematics Education, Science and Technology, London, v. 35, n. 1, p. 73-89, jan. 2004.

GRAVEMEIJER, K. Local instruction theories as means of support for teachers in reform mathematics education. Mathematical Thinking and Learning, Mahwah, v. 6, n. 2, p. 105-128, 2004.

HONG, Y.; THOMAS, M. Using the computer to improve conceptual thinking in integration. In: CONFERENCE OF THE INTERNATIONAL GROUP FOR THE PSYCHOLOGY OF MATHEMATICS EDUCATION, 21., 1997, Lahti. Proceedings... Lahti: University of Helsinki, 1997. p. $81-88$.

JOHNSON, H. L. Secondary students' quantification of ratio and rate: A framework for reasoning about change in covarying quantities. Mathematical Thinking and Learning, London, v. 17, p. 6490, ene. 2015 
KAPUT, J. J. Patterns in students' formalization of quantitative patterns. In: HAREL, G.; DUBINSKY, E. (Ed.). The Concept of Function: Aspects of Epistemology and Pedagogy, MAA Notes, volume 5. Washington: Ed. Mathematical Association of America. 1992. p. 290-318.

KOUROPATOV, A.; DREYFUS, T. Learning the integral concept by constructing knowledge about accumulation. ZDM- Mathematics Education, Berlin, v. 46, n. 2, p. 533-548, aug. 2014.

PIAGET, J. Studies in reflecting abstraction. Tradução de R. L. Campdell. Primera edición. ed. Philadelphia: Taylor \& Francis, 2001. 350 p.

RASMUSSEN, C.; BORBA, M. The teaching and learning of Calculus- In memorian Arnold Kirsh (special issue). ZDM- Mathematics Education, Berlin, v. 46, n. 2, p. 505-699, aug. 2014.

ROIG, A. I. Análisis de las fases del proceso de abstracción matemática en estudiantes de secundaria. 2008. 245 f. Tesis (Doctorado en Formación en Investigación Didáctica: Didáctica de la Matemática, Lengua y Literatura y Ciencias Sociales) - Departamento de Innovación y Formación Didáctica, Universidad de Alicante, Alicante, 2008.

ROIG, A. I.; LLINARES, S.; PENALVA, M. C. Different moments in the participatory stage of the secondary students' abstraction of mathematical conceptions. BOLEMA, Rio Claro, v. 26, n. 44, p. 1345-1366, dec. 2012.

SEALEY, V. Student understanding of definite integrals, Riemann sums and area under a curve: What is necessary and sufficient? In: NORTH AMERICAN CHAPTER OF THE INTERNATIONAL GROUP FOR THE PSYCHOLOGY OF MATHEMATICS EDUCATION, 28., 2006, Mérida. Proceedings... Mérida: Universidad Pedagógica Nacional, 2006. p. 46-53.

SIMON, M. A._Reconstructing mathematics pedagogy from a constructivist perspective. Journal for Research in Mathematics Education, Reston, v. 26, n. 1, p. 114-145, jan. 1995.

SIMON, M. A.; TZUR, R. Explicating the role of mathematical tasks in conceptual learning: An elaboration of the hypothetical learning trajectory. Mathematical Thinking and Learning, Mahwah, v. 6, n. 2, p. 91-104, 2004.

SIMON, M. A.; TZUR, R., HEINZ, K.; KINZEL, M. Explicating a mechanism for conceptual learning: Elaborating the construct of reflective abstraction. Journal for Research in Mathematics Education, Reston, v. 35, n. 5, p. 305-329, mayo 2004.

SWIDAM, O.; YERUSHALMY, M. Learning the indefinite integral in a dynamic and interactive technological environment. ZDM- Mathematics Education, Berlin, v. 46, n. 2, p. 517-531, aug. 2014.

THOMPSON, P. W. Images of rate and operational understanding of the fundamental theorem of calculus. Educational Studies in Mathematics, Dordrecht, v. 26, n. 2-3, p. 229-274, march 1994a.

THOMPSON, P. W. Students, function, and the undergraduate curriculum. In: DUBINSKY, E.; SCHOENFELD; A. H.; KAPUT, J. J. (Ed.). Research in Collegiate Mathematics Education, I: Issues in Mathematics Education volume 4. Providence: Ed. American Mathematical Society, 1994b. p. 21-44.

THOMPSON, P. W.; SILVERMAN, J. The concept of accumulation in calculus. In: M. P. CARLSON; C.; RASMUSSEN (Ed.). Making the connection: Research and teaching in undergraduate mathematics. Washington: Ed. Mathematical Association of America, 2008. p. 43-52. 
TURÉGANO, P. Del área a la integral. Un estudio en el contexto educativo. Enseñanza de las Ciencias, Barcelona, v. 16, n. 2, p. 233-249, 1998.

TZUR, R.; SIMON, M. A. Distinguishing two stages of mathematics conceptual learning. International Journal of Science and Mathematics Education, Dordrecht, v. 2, n. 2, p. 287-304, june 2004.

ZANDIEH, M. A theoretical framework for analyzing student understanding of the concept of derivative. In: DUBINSKY, E.; SCHOENFELD, A. H.; KAPUT, J. J. (Ed.). Research in Collegiate Mathematics Education, IV: Issues in Mathematics Education volume 8. Providence: Ed. American Mathematical Society, 2000. p. 103-127.

Submetido em Junho de 2016. Aprovado em Setembro de 2016. 